CrossMark $\underset{\text { \& click for updates }}{\text { Cros }}$

Cite this: J. Mater. Chem. C, 2017, 5,770

Received 6th June 2016,

Accepted 15th December 2016

DOI: $10.1039 / c 6 t c 02346 k$

www.rsc.org/MaterialsC

\section{p-Doping of polystyrene polymers with attached functional side-groups from solution $\dagger$}

\author{
Tanja Schneider, ${ }^{a}$ Felix Limberg, ${ }^{b}$ Kelvin Yao, ${ }^{c}$ Ardalan Armin, ${ }^{d}$ Nils Jürgensen, ${ }^{a}$ \\ Jens Czolk, ${ }^{a}$ Bernd Ebenhoch, ${ }^{a}$ Pascal Friederich, ${ }^{e}$ Wolfgang Wenzel, \\ Jan Behrends, ${ }^{c}$ Hartmut Krüger ${ }^{\mathrm{b}}$ and Alexander Colsmann ${ }^{* a}$
}

\begin{abstract}
Electrically doped buffer layers are often employed in organic optoelectronic devices to improve charge carrier injection or extraction. We study p-doping of non-conjugated polymers with attached hole transport moieties by intermixing strong molecular acceptors. Polymers and dopants are processed from solution and their electrical properties are benchmarked against non-polymerized, p-doped molecular materials. The equilibrium charge carrier density is estimated by electron paramagnetic resonance (EPR) measurements that show an increased number of unpaired spins upon doping. We find an increase in the conductivity of the electrically doped polymer and the corresponding molecular materials by several orders of magnitude by studying hole-only devices and employing charge extraction by linearly increasing voltage (CELIV). Altogether, the increased number of unpaired spins and the enhanced conductivity indicate the presence of free holes. The $p$-doping of the polymers with attached hole transport moieties is equally efficient as the p-doping of the corresponding molecular materials.
\end{abstract}

\section{A Introduction}

Common organic optoelectronic devices such as organic light emitting diodes (OLEDs) or organic solar cells (OSCs) are composed of photo-active layers sandwiched between two electrodes. To guarantee low-loss charge carrier injection (OLEDs) or extraction (OSCs), interface modifications or charge carrier transport layers are often implemented. Ohmic contacts with the anode can be achieved by introducing p-doped hole transport layers (HTL). To date, electrical doping of molecular systems has been extensively studied in vacuum processed organic optoelectronic devices to ensure Ohmic contacts between electrodes and functional layers. ${ }^{1-3}$ A well-established material system for efficient hole injection in vacuum processed OLEDs is $4,4^{\prime}, 4^{\prime \prime}$-tris $[$ phenyl $(m$ tolyl)amino]triphenylamine (MTDATA) p-doped with the electron acceptor 2,3,5,6-tetrafluoro-7,7,8,8-tetracyanoquinodimethane

\footnotetext{
${ }^{a}$ Light Technology Institute, Karlsruhe Institute of Technology (KIT),

Engesserstrasse 13, 76131 Karlsruhe, Germany.

E-mail: alexander.colsmann@kit.edu

${ }^{b}$ Fraunhofer Institute for Applied Polymer Research, Geiselbergstrasse 69, 14476 Potsdam, Germany

${ }^{c}$ Berlin Joint EPR Lab, Fachbereich Physik, Freie Universität Berlin, Arnimallee 14, 14195 Berlin, Germany

${ }^{d}$ Centre for Organic Photonics \& Electronics (COPE), School of Chemistry and Molecular Biosciences and School of Mathematics and Physics,

University of Queensland, 4072 Brisbane, Australia

${ }^{e}$ Institute of Nanotechnology, Karlsruhe Institute of Technology (KIT),

Hermann-von-Helmholtz-Platz 1, 76344 Eggenstein-Leopoldshafen, Germany

$\dagger$ Electronic supplementary information (ESI) available. See DOI: 10.1039/c6tc02346k
}

$\left(\mathrm{F}_{4} \mathrm{TCNQ}\right),{ }^{4,5}$ although specifically the dopant $\mathrm{F}_{4} \mathrm{TCNQ}$ may diffuse within the device at elevated temperatures. ${ }^{6}$

With respect to future low-cost roll-to-roll printing of organic devices on flexible substrates, however, there is a strong need for solution processable materials to establish Ohmic contacts with the anode. ${ }^{7}$ p-Doped poly(3,4-ethylendioxythiophene):polystyrene sulfonate (PEDOT:PSS) is widely used for hole injection or extraction in solution processed devices. Due to its hygroscopic and acidic nature, PEDOT:PSS can reduce the lifetime of optoelectronic devices, e.g., by etching the indium doped tin oxide (ITO) electrode underneath. ${ }^{8}$ Metal oxides with high work functions, ${ }^{9,10}$ or doped organic semiconductors are feasible alternatives to establish good electrical contact with the anode. ${ }^{3}$ In principle, many molecular materials that have been established in vacuum processes can be deposited from solution, too. In earlier reports, solution processed MTDATA was p-doped with 2-(3-(adamantan-1yl)propyl)-3,5,6-trifluorotetracyanoquinodimethane ( $\left.\mathrm{F}_{3} \mathrm{TCNQ}^{\mathrm{Adl}}\right)^{11}$ which showed encouraging performance but had to be synthesized in ten steps, with an overall yield below $1 \%{ }^{12}$ Other dopants, such as 1,4,5,8,9,12-hexaazatriphenylene $\left(\mathrm{HATCN}_{6}\right)$ or $\mathrm{F}_{4} \mathrm{TCNQ}$ can be synthesized in only one or two steps with overall yields of about $80 \%$ or $60 \% .{ }^{13,14}$ Molecular materials can be synthesized with higher purity than polymers, but often exhibit low viscosity, which is detrimental to many printing processes, as well as poor film forming properties upon deposition from solution due to a high tendency to form aggregates, thereby adversely affecting the film morphology and the electrical properties. The synthesis of conjugated polymers, is one way to enable the formation of 
more homogeneous films, but the extended conjugation may well change the optoelectronic properties of the functional moieties. ${ }^{15,16}$ Non-conjugated polymers with functional groups attached to the side chains often exhibit almost the same optoelectronic properties as their respective molecular counterparts, but may exhibit high glass transition temperatures and much better film forming properties. ${ }^{17,18}$

In this work, we investigated the matrices MTDATA and the corresponding non-conjugated polystyrene polymer comprising attached MTDATA-like groups (PMTDATA), p-doped with the commercially available acceptors $\mathrm{F}_{4} \mathrm{TCNQ}$ or $\mathrm{HATCN}_{6}$, processed from solution. The influence of the dopants on thin-films of MTDATA and PMTDATA were examined by electron paramagnetic resonance (EPR), near infrared (NIR) absorption spectroscopy, photoelectron yield spectroscopy in air (PESA), hole-only device currents and charge extraction by linearly increasing voltage (CELIV).

\section{B Experimental}

PMTDATA was synthesized according to processes described in the literature. ${ }^{18}$ MTDATA (Sensient), PMTDATA, HATCN $_{6}$ (Lumtec) and $\mathrm{F}_{4}$ TCNQ (97\%, Sigma Aldrich) were dissolved separately $\left(5 \mathrm{~g} \mathrm{~L}^{-1}\right)$ in tetrahydrofuran (THF, Sigma Aldrich) under inert atmosphere. The initial solutions were mixed to yield MTDATA:dopant and PMTDATA:dopant solutions with different molecular dopant concentrations.

Molecular conformations were obtained by simulations employing density functional theory (DFT) based geometry optimizations as implemented in Turbomole using a B3-LYP functional and a def2-SV(P) basis-set. ${ }^{19-21}$ Partial charges were taken from DFT calculations. Potentials for the soft innermolecular degrees of freedom (dihedral angles) were obtained using the semi-empirical PM6 method as implemented in MOPAC2012..$^{22,23}$ Atomistic morphologies containing about 1000 molecules per system were generated using the Monte Carlo (MC) based simulated annealing protocol SIMONA/DEPOSIT, ${ }^{24}$ simulating 10 annealing cycles (SA) with 150000 MC steps per molecule. The temperature was reduced from $4000 \mathrm{~K}$ to $300 \mathrm{~K}$ during each SA cycle. The ionization energies $\left(E_{\mathrm{I}}\right)$ and electron affinities $\left(E_{\mathrm{A}}\right)$ of MTDATA, $\mathrm{F}_{4}$ TCNQ, $\mathrm{HATCN}_{6}$ in mixed $(20 \mathrm{~mol} \%$ doping concentration) and pristine systems were calculated using the Quantum Patch method. ${ }^{25,26}$

The $E_{\mathrm{I}}$ of $30 \mathrm{~nm}$ thick films of undoped and doped MTDATA or PMTDATA spin coated on glass in nitrogen atmosphere were measured by PESA (beam power $10 \mathrm{nW}$ ) on an AC-2E from Riken Keiki. The absorbance spectra were measured using a Cary 5000 UV-Vis-NIR spectrophotometer from Agilent Technology under ambient conditions.

To detect unpaired spins in the doped layers, room temperature continuous wave electron paramagnetic resonance (cwEPR) spectra were recorded using a home-built X-band EPR spectrometer. Therefore, $50 \mu \mathrm{l}$ of the respective solutions were filled in EPR tubes. The solvent was removed in vacuo and the tubes were filled with inert gas and sealed using a blowtorch. The magnetic field was regulated by a field controller (Bruker BH15), and a microwave bridge (Bruker ER $048 \mathrm{R}$ ) was used for microwave generation and detection. Magnetic-field modulation in combination with lock-in detection was employed using a lock-in amplifier (Stanford Research SR810) and a modulation amplifier (Wangine WPA-120), resulting in derivative spectra. The sample was placed in a Bruker ER 4122 SHQ microwave resonator. The quality factor $Q$ of the resonator loaded with the sample was determined from the mode picture before each EPR measurement was started. The measured derivative spectra were backgroundcorrected and double integration was performed to obtain the integrated EPR signal amplitude. This intensity was compared to the double integrated intensity of a reference sample with a known number of spins (4-hydroxy-TEMPO dissolved in toluene). Taking into account $Q$, as measured for the respective sample, and all experimental parameters that influence the signal intensity, this comparison yielded the absolute number of spins. ${ }^{27}$ The uncertainty with respect to the absolute number of unpaired spins is less than $20 \%$ which is estimated based on the uncertainty of the sample volume when filling the tubes.

To measure electrical conductivities, hole-only devices with a silver $(\mathrm{Ag}, 20 \mathrm{~nm}) /$ molybdenum oxide $\left(\mathrm{MoO}_{x}, 10 \mathrm{~nm}\right) /$ matrix/ $\mathrm{MoO}_{x}(10 \mathrm{~nm}) / \mathrm{Ag}(100 \mathrm{~nm})$ architecture comprising undoped and electrically doped matrix layers were fabricated in a glovebox under nitrogen atmosphere. Therefore, glass substrates were successively cleaned in acetone and 2-propanol in an ultrasonic bath and treated with oxygen plasma. $\mathrm{Ag}$ and $\mathrm{MoO}_{x}$ were thermally evaporated through a shadow mask in an evaporation chamber $\left(\sim 10^{-6} \mathrm{mbar}\right)$ attached to the glovebox. The doped and undoped matrix solutions with varying dopant concentrations were spin coated in nitrogen atmosphere from THF (20 g L L $1000 \mathrm{rpm}, 45 \mathrm{~s}$ ) which had been stirred overnight. Current-voltage $(I-V)$ curves were recorded with a source measure unit (Keithley 238). The samples were kept under nitrogen atmosphere during the entire fabrication and characterization process. Film thicknesses were determined afterwards by a tactile stylus surface profiler (Bruker Dektak XT).

The CELIV samples employed an ITO/PEDOT:PSS/matrix/ aluminum architecture. The ITO coated glass substrates $\left(R_{\square}=13 \Omega \mathrm{sq}^{-1}\right.$ ) were cleaned using acetone and 2-propanol in an ultrasonic bath and treated with oxygen plasma to remove organic residues from the surface and to enhance PEDOT:PSS adhesion. $30 \mathrm{~nm}$ PEDOT: PSS (Clevios P VP AI 4083, Heraeus, water-diluted $1: 1$ ) was spin coated on top and annealed at $150{ }^{\circ} \mathrm{C}$ for $10 \mathrm{~min}$. The hole transport layers were processed as described above. Aluminum (200 nm) was evaporated at $\sim 10^{-6}$ mbar through a shadow mask. CELIV measurements were carried out after device encapsulation under ambient atmosphere in the dark. ${ }^{28}$ An Agilent 33522A arbitrary waveform generator was used to apply the triangular voltage pulse which was amplified to the range of $\pm 15 \mathrm{~V}$ by a Linear Technology LT1210 operational amplifier with an input impedance of $10 \mathrm{M} \Omega$. The current signal was amplified by a Femto DHPCA-100 transimpedance amplifier with a gain of $10^{4} \mathrm{~V} \mathrm{~A}^{-1}$ and recorded by an Agilent DSO 6102A digital storage oscilloscope with a load resistance of $50 \Omega$. 


\section{Results and discussion}

At the outset of our study, we investigated the main charge carrier transport levels of the matrices and dopants, which are important to describe the doping process. Fig. 1 summarizes the chemical structures of the matrices and dopants along with their ionization energies $\left(E_{\mathrm{I}}\right)$ and electron affinities $\left(E_{\mathrm{A}}\right)$. The $E_{\mathrm{I}}$ and $E_{\mathrm{A}}$ were initially computed by DFT. Here, the total energies of the molecules in their neutral and charged state were calculated. Induction and polarization effects were explicitly taken into account selfconsistently. Doing so, the individual electrostatic environment of each molecule influences $E_{\mathrm{I}}$ and $E_{\mathrm{A}}$ which are calculated as the total energy difference of a molecule in its negatively/positively charged and its neutral state. This procedure is commonly referred to as $\triangle \mathrm{SCF}$ method. For the calculation of $E_{\mathrm{I}}$ distributions of MTDATA, 5 pristine and 10 mixed morphologies were analysed. In each morphology, the innermost 100 molecules were considered. For the $E_{\mathrm{I}}$ and $E_{\mathrm{A}}$ of $\mathrm{F}_{4} \mathrm{TCNQ}$ and $\mathrm{HATCN}_{6}$, about 50 molecules from mixed and about 100 molecules from pristine morphologies were analysed. The $E_{\mathrm{I}}$ of MTDATA is shifted to lower energies if surrounded by some dopant molecules due to electrostatic effects. In a mixture comprising $20 \mathrm{~mol} \% \mathrm{~F}_{4}$ TCNQ, $E_{\mathrm{I}}$ of MTDATA is shifted from $5.07 \mathrm{eV}$ to $5.13 \mathrm{eV}$, whereas, in a mixture with $20 \mathrm{~mol}^{2} \mathrm{HATCN}_{6}, E_{\mathrm{I}}$ is shifted to $5.20 \mathrm{eV}$. Likewise, both $E_{\mathrm{I}}$ and $E_{\mathrm{A}}$ of the dopants are shifted to higher energies if surrounded by MTDATA matrix molecules. A detailed description of the simulation is provided in the ESI $\dagger$ (Section S1). In order to experimentally verify the simulated energy levels, we carried out PESA measurements on thin-films spin cast from THF solution on glass substrates. Due to the high $E_{\mathrm{I}}$ of the matrices, the determination of the energy levels in air should not lead to unintentional oxidation of the molecules by ambient oxygen and subsequent changes of the

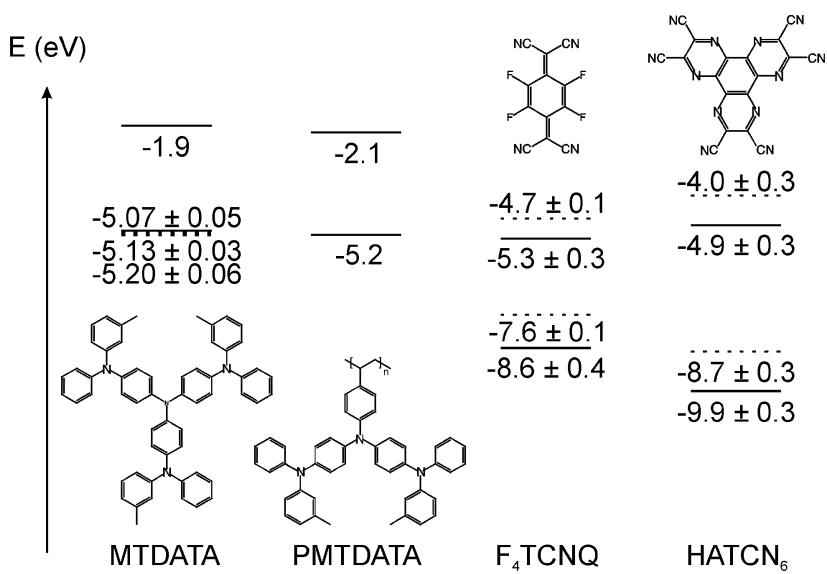

Fig. 1 Chemical structures and energy levels of the MTDATA and PMTDATA matrices as well as the $\mathrm{F}_{4} T C N Q$ and $\mathrm{HATCN}_{6}$ dopants versus vacuum $(E=0)$. The $E_{1}$ of PMTDATA was measured by PESA. $E_{1}$ and $E_{\mathrm{A}}$ of MTDATA, $F_{4} T C N Q$ and $H_{A T C N}$ were determined by DFT. When changing from pristine materials (solid lines) to matrix-dopant mixtures (20 mol\% dopant, dashed lines), according to DFT calculations, the energy levels of the dopants shift to higher energies while the energy levels of the matrix shift to lower energies.

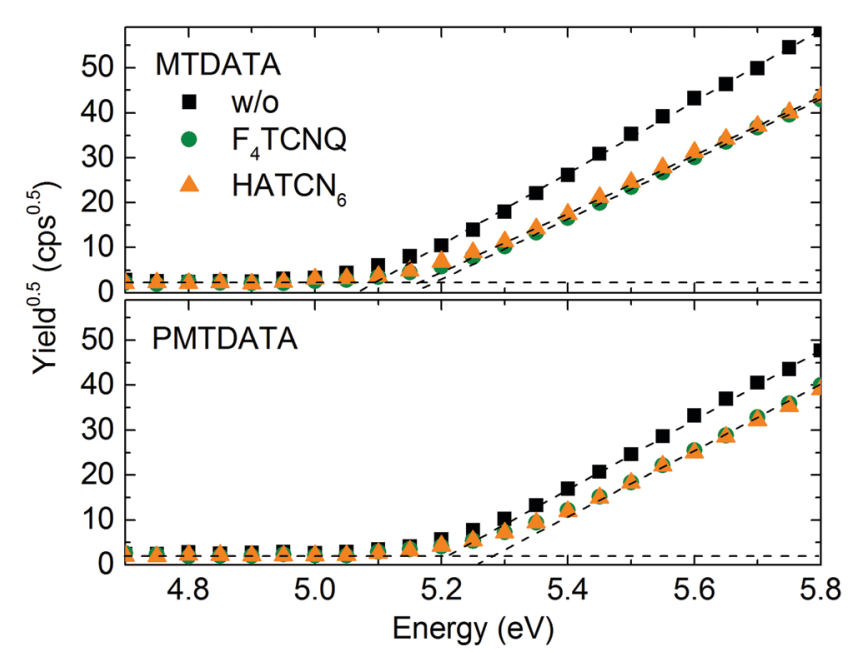

Fig. 2 Photoelectron yield of undoped and doped (20 mol\%) MTDATA and PMTDATA, measured by PESA. The shift of the ionization energy to lower energies by approximately $0.07 \pm 0.02 \mathrm{eV}$ experimentally confirms the simulated ionization energy shift due to the electrostatic interactions in the mixed system.

Table $1 E_{1}$ measured by PESA. Number of spins per volume and doping efficiency of the matrix-dopant (20 mol\%) combinations as calculated from the EPR measurements

\begin{tabular}{llll}
\hline & $E_{\mathrm{I}}(\mathrm{eV})$ & $\begin{array}{l}\text { Spin concentration } \\
\left(\mathrm{cm}^{-3}\right)\end{array}$ & $\begin{array}{l}\text { Doping } \\
\text { efficiency }(\%)\end{array}$ \\
\hline MTDATA & 5.11 & $4 \times 10^{15}$ & 0 \\
MTDATA:F $_{4}$ TCNQ $^{15}$ & 5.18 & $7 \times 10^{18}$ & 0.5 \\
MTDATA:HATCN $_{6}$ & 5.16 & $4 \times 10^{17}$ & 0.03 \\
PMTDATA $_{\text {PMTDATA:F }}$ TCNQ $^{17}$ & 5.20 & $7 \times 10^{15}$ & 0 \\
PMTDATA:HATCN $_{6}$ & 5.26 & $3 \times 10^{18}$ & 0.2 \\
PMTDA $^{18}$ & $5 \times 10^{17}$ & 0.03
\end{tabular}

energy levels. The respective measurement results are depicted in Fig. 2. The derived $E_{\mathrm{I}}$ are summarized in Table 1. We observed small shifts of the $E_{\mathrm{I}}$ of the $\mathrm{F}_{4} \mathrm{TCNQ}$ doped matrices in comparison to the undoped materials towards higher energies, $\Delta E_{\mathrm{I}}=0.07 \pm 0.02 \mathrm{eV}$ for MTDATA and $\Delta E_{\mathrm{I}}=0.06 \pm 0.02 \mathrm{eV}$ for PMTDATA, that are well in accordance with the DFT calculations above. Again, we found very similar results for both matrices, the molecular MTDATA and the polymer PMTDATA.

The doping process itself is being controversially discussed and may well depend on the actual molecular conformation. According to Gao et al., p-doping of zinc phthalocyanine with $\mathrm{F}_{4} \mathrm{TCNQ}$ is accomplished by transferring charges from the matrix molecule to the dopant. ${ }^{29}$ Pingel et al. also found an integer charge transfer upon doping of poly(3-hexylthiophene) with $\mathrm{F}_{4} \mathrm{TCNQ}^{30}$ Salzmann et al. suggested a formation of hybrid orbitals from the highest molecular orbital (HOMO) of the matrix and the lowest unoccupied molecular orbital (LUMO) of the dopant in pentacene: $\mathrm{F}_{4} \mathrm{TCNQ}$ layers, and their subsequent population by charges from the matrix' HOMO. ${ }^{31}$ In either case, free charges are generated leading to the formation of new polaronic states. ${ }^{11}$ Transitions between these states typically become visible in the absorption spectra. Fig. 3 depicts the absorption spectra of the spin coated MTDATA and PMTDATA films of the 


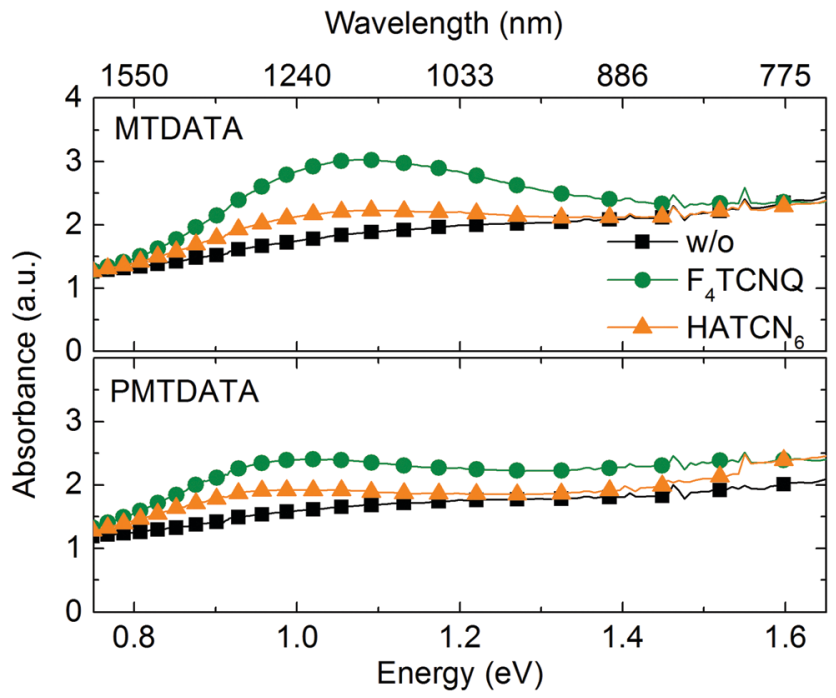

Fig. 3 Near-infrared (NIR) absorption spectra of the undoped and doped matrices MTDATA and PMTDATA (doping concentration: 20 mol\%). Absorption bands between 0.8 and $1.4 \mathrm{eV}$ indicate the formation of new polaronic states upon doping.

same doped and undoped thin-films that were used for PESA measurements, where additional absorption bands in the near infrared emerge upon p-doping of both matrices. Reference samples of undoped matrices or neat dopants do not show any absorption features in this wavelength regime. The emerging absorption band is most pronounced for MTDATA: $\mathrm{F}_{4} \mathrm{TCNQ}$ and decreases somewhat when using the host PMTDATA or the dopant $\mathrm{HATCN}_{6}$ instead.

To gain more quantitative insight into the doping mechanism, we investigated the number of unpaired electrons that form upon charge carrier transfer, in the different matrixdopant systems by EPR. Spins of unpaired electrons align along an external magnetic field and the energetic states split into two states with different magnetic spin quantum number $\left(m_{\mathrm{s}}= \pm 1 / 2\right)$. Transitions between the two energy levels can be induced by resonant microwave absorption. In a typical cwEPR experiment, field modulation in combination with lock-in detection is used, which leads to the typical derivative EPR line shape. As depicted in Fig. 4, the undoped molecular MTDATA and the polymer PMTDATA show only weak signatures of unpaired electrons. Upon p-doping of the MTDATA or PMTDATA, a strong EPR signal can be detected, indicating integer electron transfer to the dopant. To gain quantitative information of the electron transfer yield, we calculated the absolute number of spins in the samples by double-integration of the EPR signal and comparison to a 4-hydroxy-TEMPO-reference sample with a known number of spins. All measured data and the derived electrical material properties are summarized in Table 1 . Spin concentrations were calculated by dividing the total amount of measured spins by the sample volume. From the spin concentration and the density of the matrix, we determined the doping efficiency (i.e., the fraction of p-doped matrix molecules), further taking into account the paired generation of spins upon charge carrier transfer from the matrix to the dopant. Upon p-doping of MTDATA and PMTDATA

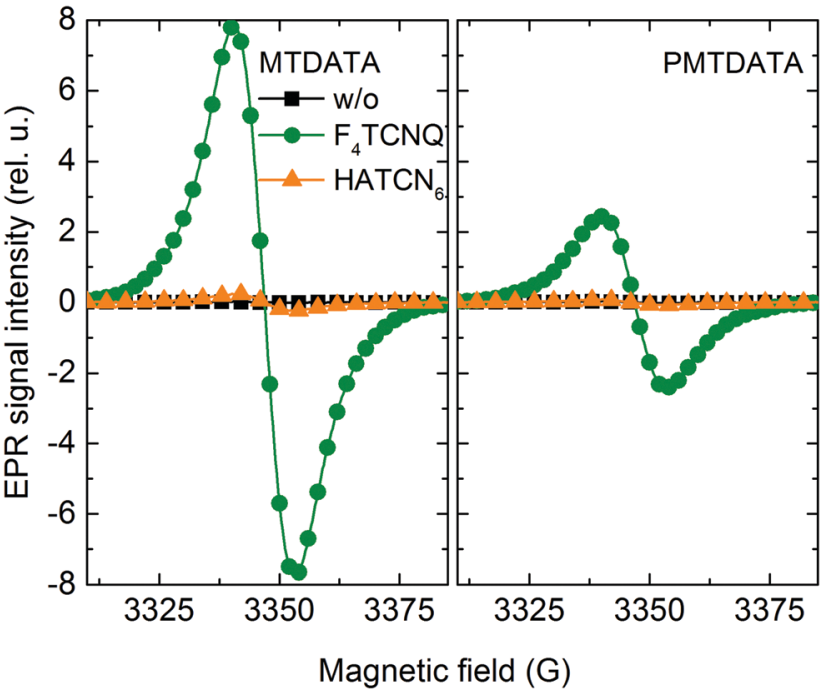

Fig. 4 EPR spectra obtained from undoped and doped (20 mol\%) MTDATA and PMTDATA. For undoped MTDATA and PMTDATA (squares), only weak EPR signals are observed. Upon doping with $\mathrm{F}_{4} T C N Q$ (circles), the EPR experiments yield a strong signature of unpaired spins and hence electrical doping. Upon doping with $\mathrm{HATCN}_{6}$, the signature is weaker (triangles), reflecting the lower $E_{\mathrm{A}}$ of $\mathrm{HATCN}_{6}$.

with $20 \mathrm{~mol} \%$ of $\mathrm{F}_{4} \mathrm{TCNQ}$, we found a substantial increase of the spin concentrations on the matrices from $4 \times 10^{15} \mathrm{~cm}^{-3}$ to $7 \times 10^{18} \mathrm{~cm}^{-3}$ and from $7 \times 10^{15} \mathrm{~cm}^{-3}$ to $3 \times 10^{18} \mathrm{~cm}^{-3}$, respectively, being well in accordance with the space charge concentrations reported in earlier work for vacuum processed MTDATA: $F_{4}$ TCNQ $\left.\left(2 \times 10^{18} \mathrm{~cm}^{-3}\right)\right)^{32}$ Importantly, the spin concentration in neat $\mathrm{F}_{4} \mathrm{TCNQ}$ that was deposited from THF solution, was two to three orders of magnitude lower. Hence we conclude that we indeed observed electrical doping of the matrix molecule rather than complex formation of THF and $\mathrm{F}_{4} \mathrm{TCNQ}$. When replacing the dopant $\mathrm{F}_{4}$ TCNQ with $\mathrm{HATCN}_{6}$, however, we observed a somewhat lower increase of the spin concentration to $4 \times 10^{17} \mathrm{~cm}^{-3}$ (MTDATA:HATCN ${ }_{6}$ ) or $5 \times 10^{17} \mathrm{~cm}^{-3}$ (PMTDATA:HATCN ${ }_{6}$ ), respectively. The respective doping efficiencies are $0.5 \%$ for MTDATA: $\mathrm{F}_{4} \mathrm{TCNQ}, 0.2 \%$ for PMTDATA: $\mathrm{F}_{4} \mathrm{TCNQ}$, and $0.03 \%$ for MTDATA:HATCN 6 and PMTDATA:HATCN 6 . Most importantly, both matrices, the molecular MTDATA and the polymer PMTDATA, yielded similar doping efficiencies. Therefore, the polymer is an excellent alternative to the molecular compound when processing p-doped organic layers from solution. The overall moderate doping efficiencies reflect the $E_{\mathrm{A}}$ of the dopants being lower than the $E_{\mathrm{I}}$ of the matrices which hampers electron transition from the matrix' HOMO to the dopant. With the $E_{\mathrm{A}}$ of $\mathrm{HATCN}_{6}$ being lower than the $E_{\mathrm{A}}$ of $\mathrm{F}_{4} \mathrm{TCNQ}$, the doping efficiency is also lower.

Electron depletion on the matrices and hence generation of equilibrium free holes are generally expected to enhance the conductivity of films. Here, the conductivities of the films versus dopant concentration were determined utilizing CELIV (in the dark) and hole-only devices as depicted in Fig. 5. Using CELIV, the conductivities were determined from the initial slope of the equilibrium charge carrier extraction current 
according to the process described in the literature, not being sensitive to the device area and thickness. ${ }^{28}$ We note that, considering film thicknesses of 400-600 nm, the influence of any depletion zones at the electrodes is negligible. ${ }^{33}$ Using holeonly devices (HODs), the hole current density $J_{\mathrm{p}}$ follows eqn (1):

$$
J_{\mathrm{p}}=J_{\mathrm{p}, \Omega}+J_{\mathrm{p}, \mathrm{SCLC}}=e p_{0} \mu_{\mathrm{p}} \frac{V}{d}+\frac{9}{8} \varepsilon_{0} \varepsilon_{\mathrm{r}} \mu_{\mathrm{p}} \frac{V^{2}}{d^{3}}
$$

with the layer thickness $d$, the charge carrier mobility $\mu$, the equilibrium charge carrier density $p_{0}$, and the dielectric constant $\varepsilon_{\mathrm{r}}$. Generally, at low voltages, the Ohmic current density $J_{\mathrm{p}, \Omega}$ determines the HODs' response to an applied voltage. As the voltage increases, the HODs are eventually driven into the space charge limited current (SCLC) regime, with $J_{\mathrm{p}, \mathrm{SCLC}}$ in eqn (1) becoming dominant. ${ }^{34}$ The HODs comprising doped layers did not reach the SCLC regime but were shunted before which did not allow for determining the charge carrier mobilities from the SCLC regime. The conductivities $\sigma_{\mathrm{p}}=e p_{0} \mu_{\mathrm{p}}$ were estimated in steady state conditions from the slope of the $J-V$ curves at low voltages where the current density follows Ohm's law (Fig. S2, ESI $\dagger)^{34,35}$ Both, MTDATA and PMTDATA, were doped with 1-15 mol\% of either dopant. The conductivities measured with both methods were in good agreement, nicely demonstrating the reliability of the experimental approaches. The conductivity of undoped MTDATA $\left(6 \times 10^{-10} \mathrm{~S} \mathrm{~cm}^{-1}\right)$ matched well the literature known conductivity of $4 \times 10^{-10} \mathrm{~S} \mathrm{~cm}^{-1}$ that was extracted from bulk $I-V$ data of vacuum deposited layers. ${ }^{2}$ With increasing doping concentration, the conductivities of all matrix-dopant combinations increased and saturates at around $10^{-7} \mathrm{~S} \mathrm{~cm}^{-1}$ above a dopant concentration of $10 \mathrm{~mol} \%$, which is again consistent with the conductivity of vacuum processed MTDATA thinfilms doped with F $_{4}$ TCNQ. $^{32}$ Although undoped PMTDATA showed a lower conductivity of $7 \times 10^{-11} \mathrm{~S} \mathrm{~cm}^{-1}$, similar conductivities were achieved in the doped systems at comparable dopant concentrations. Despite the insulating polymer backbone, the conductivity of the doped and undoped polymer PMTDATA matches the conductivities of the corresponding low-molecular MTDATA, which is well in accordance with the literature where insulating content of up to $50 \%$ does not significantly affect the electronic properties of organic semiconductors. ${ }^{36}$ To be employed as a hole injection or extraction layer in OLEDs or solar cells, respectively, the doped layer should be operated in the Ohmic regime, i.e., below the transition voltage $V_{\mathrm{T}}$ from the Ohmic to the SCLC regime. $V_{\mathrm{T}}$ can be calculated by eqn (2) that is obtained from $J_{\mathrm{p}, \Omega}=J_{\mathrm{p}, \mathrm{SCLC}}$ in eqn (1). ${ }^{35}$

$$
V_{\mathrm{T}}=\frac{8}{9} \frac{e p_{0} d^{2}}{\varepsilon_{0} \varepsilon_{\mathrm{r}}}
$$

Assuming a dielectric constant of 3.5 (estimated from the CELIV displacement current ${ }^{37}$ ) at a typical charge carrier injection layer thickness of $50 \mathrm{~nm}$ and taking into account the spin concentration from Table 1 as an upper estimate for the equilibrium hole density, we find $V_{\mathrm{T}}=46 \mathrm{mV}$ for undoped MTDATA. Hence, at typical OLED driving voltages or typical photo-voltages in solar cells, the MTDATA layer will be driven into the SCLC regime with a substantial voltage drop over the charge carrier injection layer.

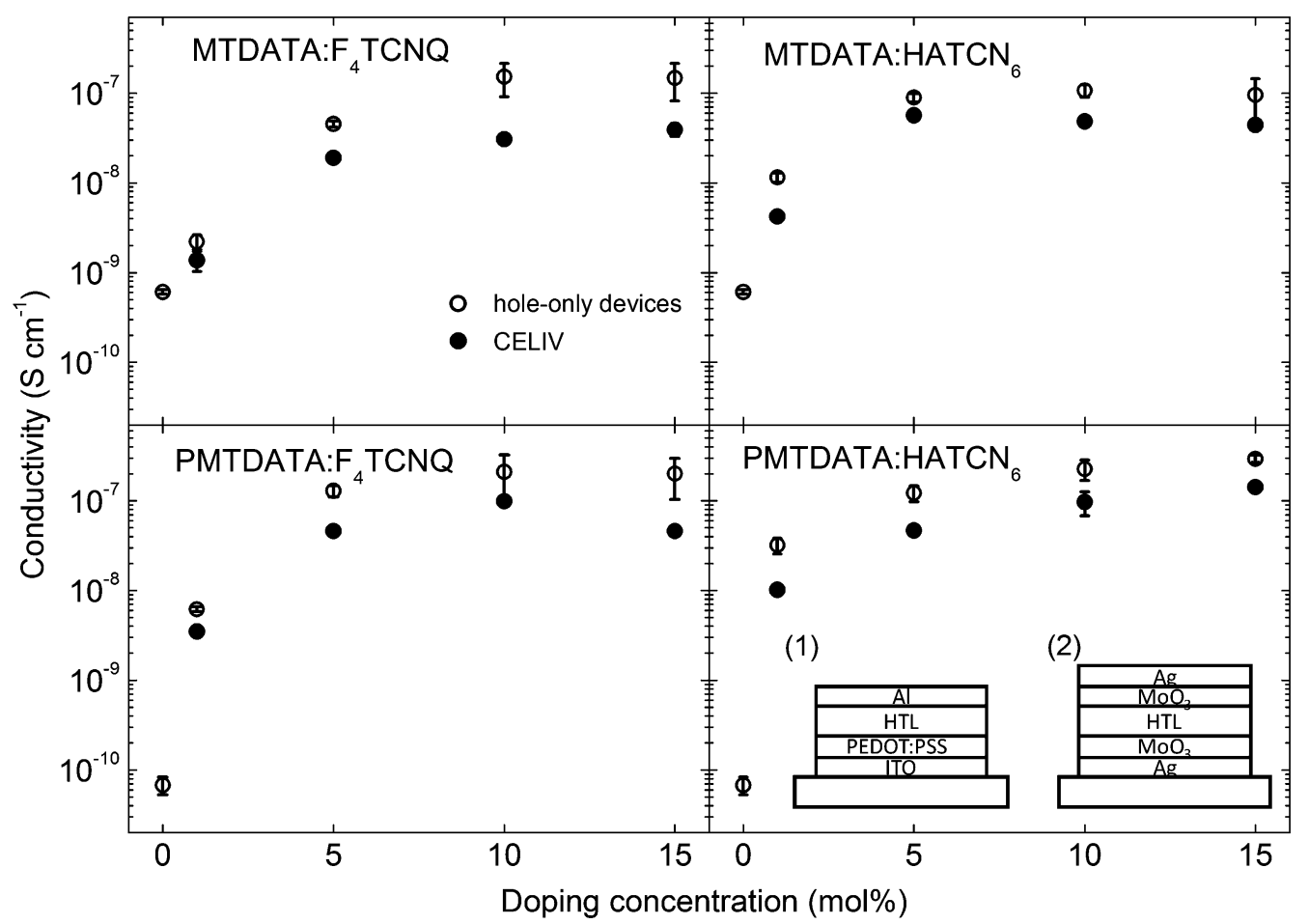

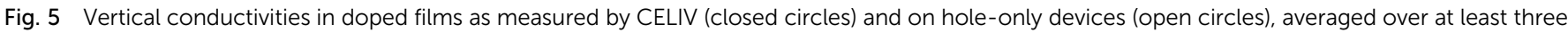

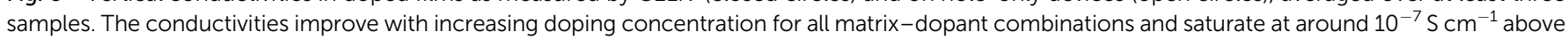
a dopant concentration of about 10 mol\%. Inset: Architectures of (1) the CELIV and (2) the hole-only devices. 
In contrast, upon p-doping with $\mathrm{F}_{4} \mathrm{TCNQ}, V_{\mathrm{T}}$ increases to $80 \mathrm{~V}$. In this case, the MTDATA: $\mathrm{F}_{4}$ TCNQ charge carrier injection layer is operated in the Ohmic regime, which implies less voltage drop over the layer and thus is more suited for device integration. For PMTDATA: $\mathrm{F}_{4}$ TCNQ, $V_{\mathrm{T}}=34 \mathrm{~V}$ is also much higher than any feasible device operating voltage, making the p-doped polymer an interesting alternative to the molecular MTDATA.

\section{Conclusions}

For both solution processable matrices, MTDATA and PMTDATA, doping with the acceptors $\mathrm{F}_{4}$ TCNQ and HATCN $_{6}$ leads to electron transfer from the matrix to the dopant. This p-doping equally enhances the electrical conductivities of the polymeric and the molecular thin-films. Our analysis shows that the achieved molecular doping levels are sufficient for designing interlayers to be employed in optoelectronic devices and are expected to form Ohmic contacts. Future printable OSCs and OLEDs may therefore rely on such solution processed p-doped charge carrier extraction or injection layers, respectively, being a viable alternative to the commonly used acidic PEDOT:PSS. With the doping of non-conjugated polymers with attached functional groups being as efficient as the doping of molecular materials, they may open a feasible way of improving film forming upon deposition from solution when using functional moieties (other than MTDATA) that tend to form aggregates.

\section{Acknowledgements}

We thank Patrick Pingel for fruitful discussions and Lars Müller for introduction to the CELIV measurement setup at the Innovation Lab (iL, Heidelberg). This work was funded by the Federal Ministry of Education and Research under contract no. 03EK3504 (project TAURUS) and the German Research Council (DFG) within SFB 1176 in the context of the project "Highly cross-linked polymers for functional multi-layers". T. S. acknowledges the Karlsruhe School of Optics \& Photonics (KSOP) for support. Simulations in this work were performed on the computational resource ForHLR I funded by the Ministry of Science, Research and the Arts Baden-Württemberg and the DFG. P.F. thanks the Carl-Zeiss Foundation for funding the project "Multiskalen Modellierung elektronischer Eigenschaften von Materialien in der organischen Elektronik".

\section{Notes and references}

1 B. Lüssem, M. Riede and K. Leo, Phys. Status Solidi A, 2013, 210, 9.

2 M. Pfeiffer, K. Leo, X. Zhou, J. S. Huang, M. Hofmann, A. Werner and J. Blochwitz-Nimoth, Org. Electron., 2003, 4, 89.

3 K. Walzer, B. Maennig, M. Pfeiffer and K. Leo, Chem. Rev., 2007, 107, 1233.

4 J. Huang, M. Pfeiffer, A. Werner, J. Blochwitz, K. Leo and S. Liu, Appl. Phys. Lett., 2002, 80, 139.
5 X. Zhou, M. Pfeiffer, J. S. Huang, J. Blochwitz-Nimoth, D. S. Qin, A. Werner, J. Drechsel, B. Maennig and K. Leo, Appl. Phys. Lett., 2002, 81, 922.

6 J. Li, C. W. Rochester, I. E. Jacobs, S. Friedrich, P. Stroeve, M. Riede and A. J. Moulé, ACS Appl. Mater. Interfaces, 2015, 7, 28420.

7 F. Nickel, T. Haas, E. Wegner, D. Bahro, S. Salehin, O. Kraft, P. A. Gruber and A. Colsmann, Sol. Energy Mater. Sol. Cells, 2014, 130, 317.

8 S. D. Yambem, K.-S. Liao, N. J. Alley and S. A. Curran, J. Mater. Chem., 2012, 22, 6894.

9 S. Höfle, H. Do, E. Mankel, M. Pfaff, Z. Zhang, D. Bahro, T. Mayer, W. Jaegermann, D. Gerthsen, C. Feldmann, U. Lemmer and A. Colsmann, Org. Electron., 2013, 14, 1820. 10 S. Höfle, M. Bruns, S. Strässle, C. Feldmann, U. Lemmer and A. Colsmann, Adv. Mater., 2013, 25, 4113.

11 J. S. Swensen, L. Wang, J. E. Rainbolt, P. K. Koech, E. Polikarpov, D. J. Gaspar and A. B. Padmaperuma, Org. Electron., 2012, 13, 3085.

12 J. E. Rainbolt, P. K. Koech, E. Polikarpov, J. S. Swensen, L. Cosimbescu, A. Von Ruden, L. Wang, L. S. Sapochak, A. B. Padmaperuma and D. J. Gaspar, J. Mater. Chem. C, 2013, 1, 1876.

13 K. Kanakarajan and A. W. Czarnik, J. Org. Chem., 1986, 51, 5241.

14 R. C. Wheland and E. L. Martin, J. Org. Chem., 1975, 40, 3101. 15 X. Gong, S.-H. Lim, J. C. Ostrowski, D. Moses, C. J. Bardeen and G. C. Bazan, J. Appl. Phys., 2004, 95, 948.

16 S. Tokito, M. Suzuki, F. Sato, M. Kamachi and K. Shirane, Org. Electron., 2003, 4, 105.

17 S. E. Shaheen, G. E. Jabbour, B. Kippelen, N. Peyghambarian, J. D. Anderson, S. R. Marder, N. R. Armstrong, E. Bellmann and R. H. Grubbs, Appl. Phys. Lett., 1999, 74, 3212.

18 M. W. Thesen, B. Höfer, M. Debeaux, S. Janietz, A. Wedel, A. Köhler, H.-H. Johannes and H. Krueger, J. Polym. Sci., Part A: Polym. Chem., 2010, 48, 3417.

19 R. Ahlrichs, M. Bär, M. Häser, H. Horn and C. Kölmel, Chem. Phys. Lett., 1989, 162, 165.

20 A. D. Becke, J. Chem. Phys., 1993, 98, 1372.

21 A. Schäfer, H. Horn and R. Ahlrichs, J. Chem. Phys., 1992, 97, 2571.

22 J. J. P. Stewart, J. Mol. Model., 2007, 13, 1173.

23 J. J. P. Stewart, MOPAC2012, Colorado Springs, 2012.

24 T. Neumann, D. Danilov, C. Lennartz and W. Wenzel, J. Comput. Chem., 2013, 34, 2716.

25 P. Friederich, F. Symalla, V. Meded, T. Neumann and W. Wenzel, J. Chem. Theory Comput., 2014, 10, 3720.

26 P. Friederich, V. Meded, F. Symalla, M. Elstner and W. Wenzel, J. Chem. Theory Comput., 2015, 11, 560.

27 G. R. Eaton, S. S. Eaton, D. P. Barr and R. T. Weber, Quantitative EPR, Springer Vienna, Vienna, 2010.

28 G. Juška, K. Arlauskas, M. Viliūnas, K. Genevičius, R. Österbacka and H. Stubb, Phys. Rev. B: Condens. Matter Mater. Phys., 2000, 62, 235.

29 W. Gao and A. Kahn, Appl. Phys. Lett., 2001, 79, 4040.

30 P. Pingel and D. Neher, Phys. Rev. B: Condens. Matter Mater. Phys., 2013, 87, 115209. 
31 I. Salzmann, G. Heimel, S. Duhm, M. Oehzelt, P. Pingel, B. M. George, A. Schnegg, K. Lips, R.-P. Blum, A. Vollmer and N. Koch, Phys. Rev. Lett., 2012, 108, 035502.

32 J. Drechsel, M. Pfeiffer, X. Zhou, A. Nollau and K. Leo, Synth. Met., 2002, 127, 201.

33 T. Kirchartz, W. Gong, S. A. Hawks, T. Agostinelli, R. C. I. MacKenzie, Y. Yang and J. Nelson, J. Phys. Chem. C, 2012, 116, 7672 .
34 A. Armin, G. Juska, B. W. Philippa, P. L. Burn, P. Meredith, R. D. White and A. Pivrikas, Adv. Energy Mater., 2013, 3, 321. 35 Y. Zhang, B. De Boer and P. W. M. Blom, Adv. Funct. Mater., 2009, 19, 1901-1905.

36 A. D. Scaccabarozzi and N. Stingelin, J. Mater. Chem. A, 2014, 2, 10818.

37 Q. Lin, A. Armin, R. C. R. Nagiri, P. L. Burn and P. Meredith, Nat. Photonics, 2015, 9, 106. 